\title{
Radio Resource Management for OFDMA-Based Mobile Relay Enhanced Heterogenous Cellular Networks
}

\author{
İlhan BAŞTÜRK*, Berna ÖZBEK*, Çağatay EDEMEN ${ }^{\dagger}$, Ahmet Serdar TAN ${ }^{\ddagger}$, Engin ZEYDAN ${ }^{\S}$ and Salih ERGÜT ER $^{\S}$ \\ *Electrical and Electronics Engineering Department, Izmir Institute of Technology, Izmir, Turkey, \\ Email: ilhanbasturk,bernaozbek@iyte.edu.tr \\ $\dagger$ Electrical and Electronics Engineering Department, Işık University, Istanbul, Turkey, \\ Email: cagatayedemen@isikun.edu.tr \\ ${ }^{\ddagger}$ Türk Telekomünikasyon A.Ş., Istanbul, Turkey \\ Email: ahmetserdar.tan@turktelekom.com.tr \\ $\S$ AveaLabs, Istanbul, Turkey \\ Email: engin.zeydan,salih.ergut@avea.com.tr
}

\begin{abstract}
In this paper, we focus on the radio resource management problem for the Orthogonal Frequency Division Multiple Access (OFDMA)-based mobile relay-enhanced heterogenous cellular networks. We combine mobile relaying and data offloading scenarios to increase the capacity of the system and cope with the mobile data traffic volume that is increased by the number of wireless subscribers accessing mobile data services. We propose network interface selection, relay selection and resource allocation solutions for this scenario and show effect of relaying and data offloading on the system capacity and on the ratio of satisfied users.
\end{abstract}

\section{INTRODUCTION}

One of the key expectations for the future wireless system is to provide ubiquitous high data rate coverage in the most cost-effective manner. During the recent years, a large part of the research has focused on OFDMA transmission technology, a very promising candidate for the physical layer in next generation cellular system, due to its inherent robustness against frequency-selective fading and its capacity for achieving high spectral efficiency. In OFDMA, each subcarrier can be allocated to a different user which can best exploit the current channel condition, hence maximizing the achievable capacity. However, with the traditional cellular architecture, increasing the capacity along with the coverage would require the deployment of a large number of Base Stations (BS), which turns out to be a cost-wise inefficient solution to service providers. However, introducing Relay Stations (RS) in each cell can alleviate this problem since the RS can forward high data rates in remote areas of the cell while keeping a low cost of infrastructure [1]. In the literature two different types of relaying network architecture have been investigated as fixed relay station (FRS) and mobile relay station (MRS) which can be two types such as MRSs fitted on moving vehicle and the other is the Mobile Stations (MS) acting as MRSs [2]. The FRSs are part of the network infrastructure, thus where and how much FRSs will be deployed in a cell will be processed while the network planning, design and deployment process by operators. Compared to FRS, MRS can be flexible employed in a wireless cellular network. The goal of employing MRSs is not to replace FRSs, but rather to act as a complementary solution. The usage of MRSs to extend coverage and to increase throughput has been studied in [3] and the results indicated an increase in coverage and in maximum throughput.

In these relay-enhanced networks, potential gain in capacity and coverage is highly dependent on the radio resource management (RRM) strategy [4][5], a topic which draws more and more attention of the research community. How to perform RRM in such a complex environment is a big challenge since increased number of links makes the resource allocation problem difficult to tackle. Many prior studies focused on solving this problem for different scenarios such as downlink/uplink single cell or multicell by using different constraints such as fairness, load balancing, traffic awareness for the fixed relay networks (FRNs) [6]-[13]. A downlink single-cell network with a single fixed RS is considered in [6], while such a network with multiple fixed RSs is studied in [7]. The subcarrier and power allocation scheme that can guarantee load fairness among relays is given in [8]. In [9] and [10] resource allocation algorithms are proposed to maximize the sum rate in OFDMA multihop relaying downlink single-cell scenario. A fair traffic-awareness resource allocation scheme that can significantly reduce the co-channel interference and improve spectrum utilization in OFDMA-based multicellular networks enhanced by fixed relays is presented in [11] and [12]. In [13], any fairness constraint is not considered and its tendecy is to maximize the total cell capacity for multicellular networks. The RRM literature for mobile relay networks (MRNs) is not as rich as FRNs. In [14], it is proposed to maximize the uplink system throughput subject to the distributed power and queue state constraints of each user which has the ability to relay information to each other to improve the overall system performance.

Furthermore, cellular networks are overloaded with mobile data traffic due to the rapid growth of mobile broadband subscriptions. Future wireless networks are expected to combine multiple access technologies and work heterogeneously in order to distribute the traffic load among these networks. Of- 
floading traffic from the primary access technology to another access technology when applicable provide extra capacity and improve overall performance [15].

In this paper, we focus on an OFDMA-based mobile relay-enhanced RRM problem for the heterogenous cellular networks. In the proposed system, Long Term Evolution (LTE) based macro BS is used as a primary access network and OFDMA integrated WLAN access points (APs) are used as the secondary access network. A network interface selection algorithm is proposed that offloads some of the users from macro BS to APs in order to prevent overloading of the network. The resource allocation problem is handled disjointly for the macro and AP users. Cell-edge users suffer a reduction in data rates as compared with users which are close to the BS because their Signal to Noise Ratio (SNR) is usually lower. This causes some unfairness among nodes. The use of relays decreases the transmission range, increasing the probability of receiving the data correctly, allowing higher data rates than those achieved without relays. Thus, we integrate mobile relaying concept into this heterogenous cellular network structure to increase the number of satisfied users and to increase the capacity of the cell-edge users' in the network. Some of the mobile users that are close to the BS are used as mobile relays for the users which are far from the BS. The gain of using mobile relaying and data offloading over the number of satisfied users and data rate of cell-edge users is shown through simulation results.

\section{SySTEM MODEL}

In this paper, a single cell downlink OFDMA-based mobile relay-enhanced topology is used as shown in Figure 1. The BS is located in the centre of the cell and $K$ users are distributed uniformly around it. The cell area is divided into two zones; inner and outer. Let $d$ denote the distance of a user at point $(x, y)$ to the $\mathrm{BS}$ at the origin $(0,0)$ and $R$ is the radius of the cell. Then the inner and outer zones represents the regions where $d \leq \rho R$ and $d>\rho R$, respectively, $0<\rho<1$. The term $\rho$ defines the boundary between the zones. In Figure 1, the zone boundary is plotted for $\rho=2 / 3$. Moreover, $W$ APs are located at the cell edge in order to relieve the heavy traffic load. It is allowed that the users which are in the inner zone use LTE network and communicate the BS directly. The users which are in the outer zone can use not only LTE but also OFDMA integrated WLAN network. If LTE network is chosen, they are allowed to communicate with the BS either directly or over another user (mobile relaying). We can determine the cell edge users' mobile relay candidates using the area whose radius is $R / 2$.

The system resources for both networks are disjointly allocated among the users. Resource allocation is performed by assuming that the channel state information (CSI) of related users is available at the APs and macro BS. In this study, Half Duplex (HD) relaying is used in which each RS operates in two phases; BS and RS subframes. Resource allocation is performed in two time slots, which has equal duration, as shown in Figure 2. In the first time slot called BS subframe, BS sends data to MSs and RSs and in the second time slot called RS subframe, MSs receive information from RSs and BS. Subchannels are allocated to the $\mathrm{BS} \rightarrow \mathrm{MS}, \mathrm{BS} \rightarrow \mathrm{RS}$ links at time slot one and $\mathrm{RS} \rightarrow \mathrm{MS}, \mathrm{BS} \rightarrow \mathrm{MS}$ links at time slot two.

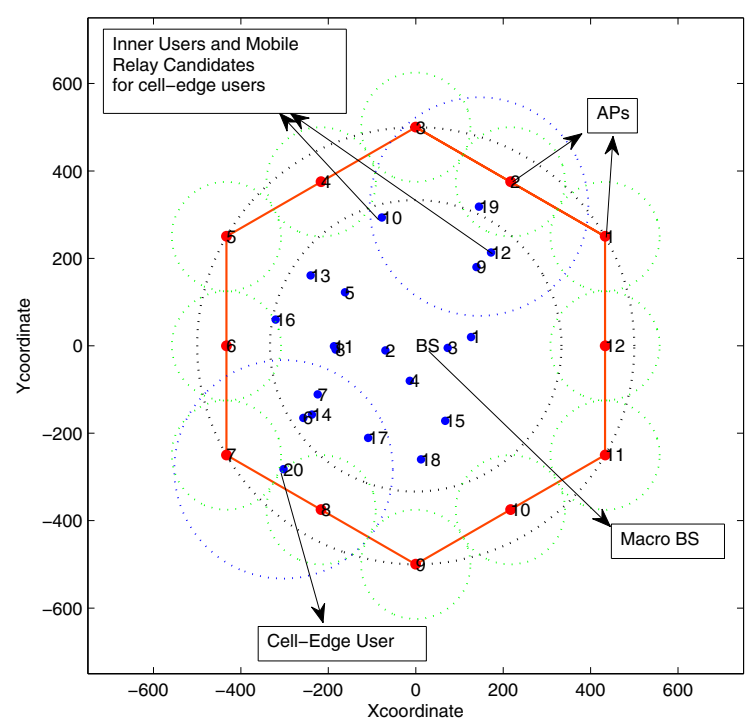

Fig. 1: System model for heteregenous wireless network.

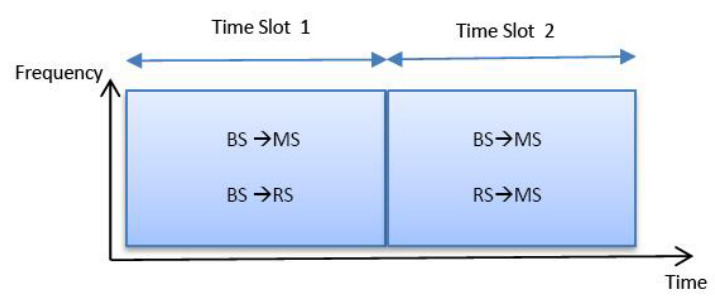

Fig. 2: HD relaying slot structure for LTE.

\section{THE PROPOSED RRM FOR MOBILE RELAY-ENHANCED HETEREGONEOUS NETWORKS}

The number of mobile users is increasing significantly and it is getting harder to satisfy all users' Quality of Service (QoS) requirements. It is mandatory to find solutions to increase the capacity and satisfy more users as much as possible. Thus, we combine two scenarios such as mobile relaying and data offloading and propose RRM solution as network interface selection, relay selection and resource allocation.

In such multi-access wireless access networks, network discovery and access selection are the fundamental problems. As its name indicates, access selection refers to the process of deciding over which access network to connect at any point in time. In this study, a new metric called network interface quality metric (IQM) is proposed to select the best network interface. The value of this metric varies between 0 and 1 and it is calculated by multiplying the subband data rate, the normalization constant and the number of available subbands on each interface or medium. The IQM metric for $k t h$ user for $i t h$ interface at frame $m$ is calculated as:

$$
I Q M_{k, i}(m)=\gamma_{i} \sum_{q=1}^{Q_{i}} A W B_{i, q}(m-1) \eta_{k, i, q}(m)
$$


where $Q_{i}$ is the total number of subbands for interface $i \in\left\{A P_{k}, L T E\right\}, A W B_{i, q}(m-1)$ is the indicator that shows the availability of $q$ th subband for interface $i$ at frame $m-1$ when it is equal to 1 and 0 otherwise. $\eta_{k, i, q}(m)$ is the data rate for the $k t h$ user for subband $q$ at ith interface at frame $m . \gamma_{i}=\frac{1}{Q_{i} \times \eta_{i}^{\text {max }}}$ is the normalization constant and $\eta_{i}^{\text {max }}$ is the maximum supportable data rate value of one subband at interface $i$. The normalization constant is required due to different supportable data rate of the interfaces. Therefore, it gives us a fair comparison between interfaces. The proposed network interface selection algorithm that uses the IQM metric is given in detail as follows:

\section{I.Interface Selection Algorithm}

- Let $\mathbb{O}$ be outer user set and $\mathbb{W}$ is the APs set.

for $\forall k \in \mathbb{O}$ do

* Find the APs that is in the coverage area of user $k$

$$
\Upsilon_{k}=\left\{w \mid d_{k, w} \leq d_{t h}, w \in \mathbb{W}\right\}
$$

* If $\Upsilon_{k} \neq \emptyset$, calculate the $S N R_{k, w}=\frac{P_{k, w}}{N_{0} B}, \forall w \in \Upsilon_{k}$

* Find the best AP candidate for user $k$ that satisfies;

$$
w^{\prime}=\arg \max _{\forall w \in \Upsilon_{k}}\left(S N R_{k, w}\right),
$$

* If $S N R_{k, w^{\prime}}>S N R_{t h}$, the AP for user $k$ is $A P_{k}=w^{\prime}$

$*$ Calculate the IQM metric for all interfaces using (1).

$*$ Select the interface for user $k$

$$
I N T_{k}= \begin{cases}W L A N, & \text { if } I Q M_{k, A P_{k}}(m) \geq I Q M_{k, L T E}(m) \\ L T E, & \text { o.w. }\end{cases}
$$

end for

where $d_{k, w}$ is the distance and $P_{k, w}$ is the average received power, which is determined by the transmitted power and the path loss, shadowing, and multipath fading, between AP $w$ and cell edge user $k . d_{t h}$ and $S N R_{t h}$ is the threshold distance and SNR value that is used to decide the AP candidates. $N_{0}$ is the noise power and $B$ is the total bandwidth.

The basic idea in practical system is whenever an AP is available, some or all of the traffic is routed through the AP, thus offloading the cellular access. However, this strategy can not be efficient since the APs may be over-crowded which means all bands can be fully utilized. Thus, the proposed interface network selection algorithm consider the bandwidth availability parameter that prevents routing a user to overloaded network.

After the selection of access networks for cell-edge users, relay selection and resource allocation is performed for macro BS users and resource allocation is performed for AP users. Resource allocation algorithm for the AP users are given in detail below;

\section{II.Resource Allocation Algorithm for AP Users}

- Let $\mathbb{W}, K_{w}, N^{\prime}, R_{k}, R_{t h}, \mathbb{U}_{w}$ be the set of all APs in the cell, the number of users that selected the AP $w$, the number of subbands, data rate of the kth user belongs to AP $w$, the threshold data rate that each user has to get and unsatisfied user set of AP $w$, respectively.

for $\forall w \in \mathbb{W}$ do

$* \mathbb{U}_{w}=\left\{1,2, \ldots, K_{w}\right\}, R_{k}=0 \forall k \in \mathbb{U}_{w}, n=1$

While $\mathbb{U}_{w} \neq \emptyset$ do

* Find the Modulation and Coding Scheme (MCS) values for $\forall k \in \mathbb{U}_{w}$

$$
M C S_{k, n}=M C S_{w \rightarrow k, n}
$$

* Find the user $k^{\prime}$ that has the maximum MCS value for the subband $n$,

$$
k^{\prime}=\arg \max _{k \in \mathbb{U}_{w}}\left(M C S_{k, n}\right)
$$

* Update the $R_{k^{\prime}}$ by adding the MCS value that are already given to that user.

* If $R_{k^{\prime}} \geq R_{t h}$, user $k^{\prime}$ is satisfied, so remove it from the unsatisfied user set $\mathbb{U}_{w} \leftarrow \mathbb{U}_{w} \backslash k^{\prime}$.

* Increase $n$ by 1 until $n=N^{\prime}$.

end while

end for

MCS values are determined using MCS table of $802.11 \mathrm{n}$ standard [16].

The relay selection and resource allocation algorithms for LTE based macro BS users can be given as follows:

\section{III.Relay Selection and Resource Allocation for LTE Users}

\section{A-Relay Selection Algorithm}

- Let $\mathbb{O}_{L T E}$ and $\mathbb{I}_{L T E}$ be outer and inner user sets that selected LTE network, respectively

for $\forall l \in \mathbb{O}_{L T E}$ do

* If exist, find a relay candidate set for user $l$

$$
\zeta_{l}=\left\{j \mid d_{l, j} \leq d_{t h}, j \in \mathbb{I}_{L T E}\right\}
$$

* If $\zeta_{l} \neq \emptyset$, choose one relay through the relay candidates using the Minimum Total Pathloss (MTP) Selection [17],

$$
r_{l}=\arg \min _{j \in \zeta_{l}}\left(P L_{B S \rightarrow j}+P L_{j \rightarrow l}\right) .
$$

\section{end for}

where $j$ is the any inner user, $\zeta_{l}$ is the set of relay candidates of outer LTE user $l, d_{l, j}$ is the distance between inner user $j$ and outer user $l$ and $d_{t h}$ is the threshold distance that is used to decide the relay candidates. $P L_{B S \rightarrow j}$ and $P L_{j \rightarrow l}$ denote the pathlosses in $\mathrm{dB}$ associated with the first (between the $\mathrm{BS}$ to the candidate relaying node) and the second (between the candidate relaying node to the relayed node) hops, respectively. The selected relay candidates are sent to the BS by the cell-edge users and BS uses this information for the resource allocation.

\section{B-Resource Allocation Algorithm}

- Let $\mathbb{N}_{1}, \mathbb{N}_{2}$ be the set of subchannels at time slot 1 and 2 , respectively. $K_{L T E}, \mathbb{U}_{L T E}$ are the total number of LTE users 
and set of unsatisfied LTE users.

- Initially, $\mathbb{U}_{L T E}=\left\{1,2, \ldots, K_{L T E}\right\}, R_{k}=0, \forall k \in \mathbb{U}_{L T E}$, $\mathbb{N}_{1}=1,2, \ldots, N, \mathbb{N}_{2}=1,2, \ldots, N, n=1$

Step1

While $\mathbb{U}_{L T E} \neq \emptyset$ do

* Determine the $C Q I_{k, n}$, Channel Quality Indicator (CQI) for $\forall k \in \mathbb{U}_{L T E}$

if $k \in \mathbb{O}_{L T E}$ and $r_{k} \neq \emptyset$ do

$C Q I_{B S \rightarrow r_{k} \rightarrow k, n}=\min \left\{C Q I_{B S \rightarrow r_{k}, n}, C Q I_{r_{k} \rightarrow k, n}\right\}$

$C Q I_{k, n}=\max \left\{C Q I_{B S \rightarrow k, n}, C Q I_{B S \rightarrow r_{k} \rightarrow k, n}\right\}$

else do

$C Q I_{k, n}=C Q I_{B S \rightarrow k, n}$

end if

* Find the user $k^{\prime \prime}$ that has the maximum CQI value for the subchannel $n$,

$$
k^{\prime \prime}=\arg \max _{k \in \mathbb{U}_{L T E}}\left(C Q I_{k, n}\right)
$$

* Update the $R_{k^{\prime \prime}}$ using CQI table.

* If $R_{k^{\prime \prime}} \geq R_{t h}$, user $k^{\prime \prime}$ is satisfied, so remove it from the unsatisfied user set, $\mathbb{U}_{L T E} \leftarrow \mathbb{U}_{L T E} \backslash k^{\prime \prime}$.

* If user $k^{\prime \prime}$ is a user that communicates with BS over a relay, remove the subchannel $n$ from the second time slot subchannel set. Since, we assume that the RS transmit and receive at the same subchannel, $\mathbb{N}_{2} \leftarrow \mathbb{N}_{2} \backslash n$

* Increase $n$ by 1 until $n=N$.

end while

Step2

While $\mathbb{U}_{L T E} \neq \emptyset$ and $\mathbb{N}_{2} \neq \emptyset$ do

* Select a subchannel $n$ through the remaining subchannels of $\mathbb{N}_{2}$

* Determine the CQI values for $\forall k \in \mathbb{U}_{L T E}$ at subchannel $n \in \mathbb{N}_{2}$

$$
C Q I_{k, n^{\prime}}=C Q I_{B S \rightarrow k, n^{\prime}}
$$

* Find the user $k^{*}$ that has the maximum CQI value for the subchannel $n^{\prime}, k^{*}=\arg \max _{k \in \mathbb{U}_{L T E}}\left(C Q I_{k, n^{\prime}}\right)$

* Update the $R_{k *}$ using CQI table.

* If $R_{k^{*}} \geq R_{t h}$, user $k^{*}$ is satisfied, so remove it from the unsatisfied user set $\mathbb{U}_{L T E} \leftarrow \mathbb{U}_{L T E} \backslash k^{*}$.

$* \mathbb{N}_{2} \leftarrow \mathbb{N}_{2} \backslash n^{\prime}$

\section{end while}

In the algorithms, CQI values are determined using LTE CQI table [18]. Maximum CQI scheduling in which the subchannel is given to the link which has the maximum CQI value is used to allocate the resources to the users. In order to obtain fairness between the users, a threshold data rate value $R_{t h}$ is determined and the user that reached this data rate is removed from the system and called as satisfied user.

\section{PERFORMANCE RESUlTS}

In the performance evaluation, we have used the simulation parameters summarized in Table I and Table II for macro BS and AP users, respectively.
TABLE I: Simulation Parameters for macro BS users

\begin{tabular}{|l|l|}
\hline \hline Parameter & Value \\
\hline Frequency & $2 \mathrm{GHz}$ \\
\hline Bandwidth & $20 \mathrm{MHz}$ \\
\hline Thermal Noise Density & $-134.89 \mathrm{dBm} / \mathrm{Hz}$ \\
\hline nTX $\times$ nRX antennas & $1 \times 1$ \\
\hline eNodeB TX power & $49 \mathrm{dBm}$ \\
\hline UE as relay TX power & $23 \mathrm{dBm}$ \\
\hline Cell radius & $500 \mathrm{~m}$ \\
\hline Pathloss model & $\mathrm{BS} \rightarrow \mathrm{MS}, \mathrm{BS} \rightarrow \mathrm{RS}$ and RS $\rightarrow \mathrm{MS}$ \\
& $128.1+37.6 \log 10(d)$ \\
\hline Shadowing model & Lognormal distribution, $\mu=0, \sigma=10(d B)$ \\
\hline Multipath model & Extended Pedesterian A (EPA) \\
\hline \hline
\end{tabular}

TABLE II: Simulation Parameters for AP users

\begin{tabular}{|l|l|}
\hline \hline Parameter & Value \\
\hline Frequency(fc) & $2.4 \mathrm{GHz}$ \\
\hline Bandwidth & $20 \mathrm{MHz}$ \\
\hline Thermal Noise Density & $-134.89 \mathrm{dBm} / \mathrm{Hz}$ \\
\hline nTX $\times$ nRX antennas & $1 \times 1$ \\
\hline AP TX power & $20 \mathrm{dBm}$ \\
\hline Pathloss model & $20 \log 10\left(\frac{4 \pi f c d B P}{3 e 8}\right)+35 \log 10\left(\frac{d}{d_{B P}}\right)$ \\
& $d_{B P}: 10$ (breakpoint distance in meters) \\
\hline Shadowing model & Lognormal distribution, $\mu=0, \sigma=5(d B)$ \\
\hline Multipath model & IEEE 802.11 TGn channel model D \\
\hline Number of APs & 12 \\
\hline \hline
\end{tabular}

The cell radius is chosen as $R=500 \mathrm{~m}$ and the users which are far from the $\rho R$ where $\rho$ is chosen $2 / 3$ in the cell is labeled as the cell-edge users. The percentage of cell-edge users is chosen as $10 \%$. The coverage area radius $d_{t h}$ is chosen $250 \mathrm{~m}$ to find the APs and relay candidates for the cell-edge users. Moreover, $S N R_{t h}$ is set to $0 d B$ in order to determine the AP of a cell-edge user.

In the simulation results, we have compared four different cases; all users are connected to macro BS directly (macro case), some cell edge users are using mobile relays for communication (macro+relay case) or using APs for communication (macro+ap case) and outer users can access macro BS or AP and also use mobile relays if their direct links are not good enough (macro+ap+relay case). These cases are compared in terms of percentage of satisfied users and sum rate of cell-edge users. The simulation results are obtained for $R_{t h}=168 \mathrm{kbps}$.

In Figure 3, the percentage of satisfied users are compared and it is shown that the number of satisfied users are increased not only by using mobile relays (macro+relay) but also offloading data to the APs (macro+ap) compared to only macro case. The number of satisfied user is reached to the best level compared to other cases when combining mobile relaying and data offloading (macro+ap+relay).

Sum data rate of cell-edge users is given in Figure 4. When the mobile relays and APs are used in the system, the cell edge data rate is being increased. Results showed an increase in the cell-edge capacity ranging from $9 \%$ to $16 \%$ by using mobile relays and $5.3 \%$ to $7 \%$ by using APs depending on the number of users. In addition to macro+relay and macro+ap cases, combination of mobile relaying and data offloading also increased cell-edge capacity ranging from $13.6 \%$ to $20.6 \%$ depending on the number of users. macro+ap+relay case is the best among all since offloading some of the data is relieved the LTE network traffic and increased the user capacity. 


\section{CONCLUSION}

In this paper, we have developed a solution for RRM problem of the OFDMA-based mobile relay-enhanced heterogeneous cellular networks. We have revealed that using mobile relays and data offloading scenarios with effective RRM algorithms as network interface selection, relay selection and resource allocation, the cell-edge user data rate and the number of satisfied users increase in a network. The proposed interface network selection algorithm that consider the bandwidth availability parameter prevented routing a user to overloaded network which decrease the data rate of the cell-edge user.

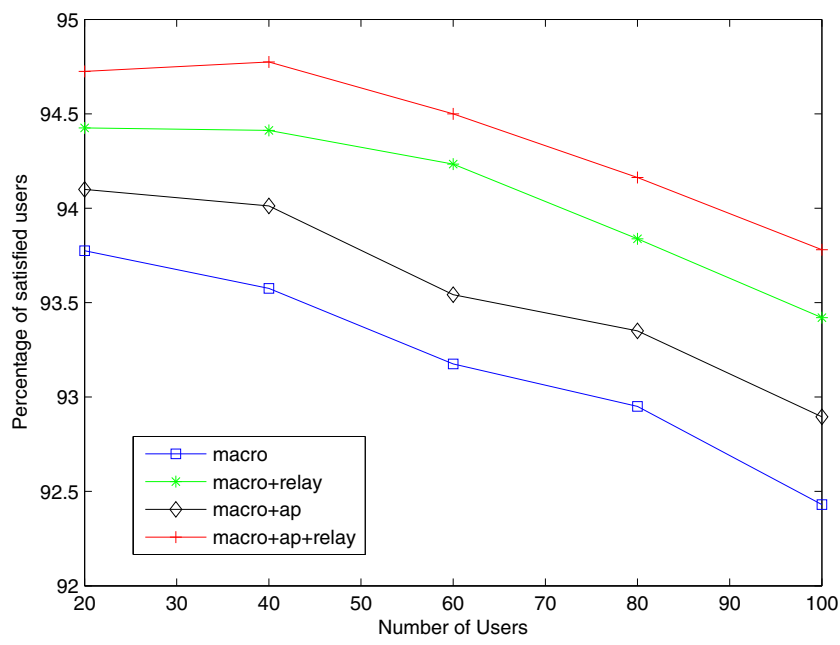

Fig. 3: Percentage of satisfied users vs the number of users.

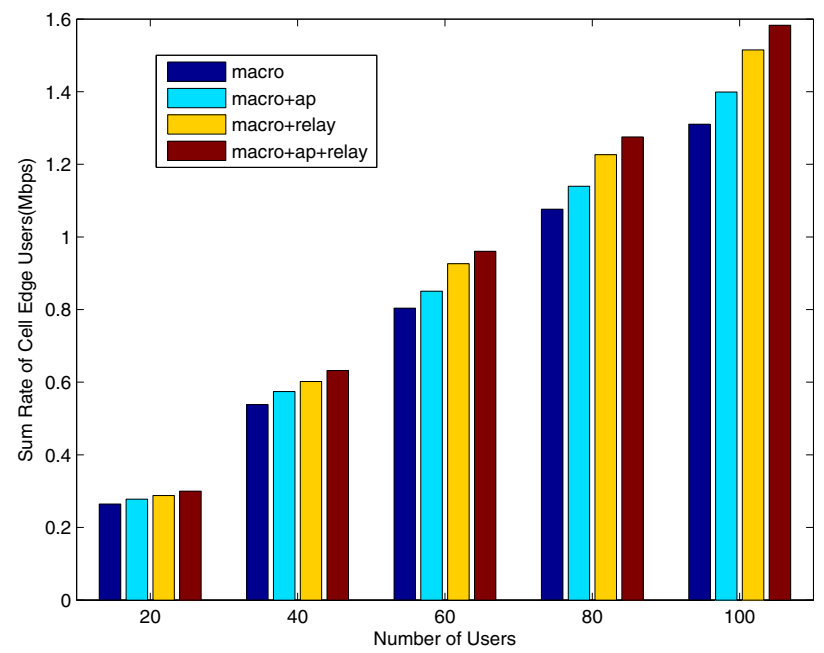

Fig. 4: Sum rate of cell edge users vs the number of users.

\section{ACKNOWLEDGMENT}

This work has been performed in the framework of the CELTIC project CP7-011 MEVICO.

\section{REFERENCES}

[1] R. Pabst et al., "Relay-Based Deployment Concepts for Wireless and Mobile Broadband Radio," IEEE Wireless Comm. Mag., vol.42, pp. 80-89, September 2004.

[2] H. Nourizadeh, S. Nourizadeh and R. Tafazolli, "Performance evaluation of Cellular Networks with Mobile and Fixed Relay Station," IEEE VTC, pp.1-5, September 2006.

[3] L Xiao, TE Fuja, DJ Costello, "Mobile relaying: coverage extension and throughput enhancement". IEEE Transactions on Communications, vol.58, pp. 2709-2717, 2010.

[4] M. Salem, A. Adinoyi, H. Yanikomeroglu, and D. Falconer, "Opportunities and challenges in OFDMA-based cellular relay networks: A radio resource management perspective," IEEE Trans. Veh. Technol., vol. 59, pp. 2496-2510, 2010.

[5] M. Salem et al., "An Overview of Radio Resource Management in Relay-Enhanced OFDMA-Based Networks," IEEE Comm. Surveys Tutorials, vol. 12, no. 3, pp. 422-438, Third Quarter 2010.

[6] M. Kaneko and P. Popovski, "Radio resource allocation algorithm for relay-aided cellular OFDMA system," IEEE ICC, pp. 4831-4836, June 2007.

[7] W. Nam, W. Chang, S.-Y Chung, and Y. Lee, "Transmit optimization for relay-based cellular OFDMA systems," IEEE ICC, pp. 5714-5719, June 2007.

[8] C. Bae and D.H. Cho, "Fairness-aware adaptive resource allocation scheme in multi-hop OFDMA systems," IEEE Commun. Lett., vol.11, no.2, pp. 134-136, Feb. 2007.

[9] R. Kwak and J. M. Cioffi, "Resource-allocation for OFDMA multihop relaying downlink systems," IEEE GLOBECOM, pp. 3225-3229, Nov. 2007.

[10] L. Huang, M. Rong, L. Wang, Y. Xue, and E. Schulz, "Resource allocation for OFDMA based relay enhanced cellular networks," IEEE VTC, pp. 3160-3164, April 2007.

[11] M. Salem, A. Adinoyi, M. Rahman, H. Yanikomeroglu, D. Falconer, Y.- D. Kim, and E. Kim, "Fairness-aware joint routing and scheduling in OFDMA-based multi-cellular fixed relay networks," IEEE ICC, pp. 1-6, June 2009.

[12] M. Salem, A. Adinoyi, H.Yanikomeroglu, et al, "A Fair Radio Resource Allocation Scheme for Ubiquitous High-data-rate Coverage in OFDMA based Cellular Relay Networks," IEEE GLOBECOM, pp. 1-6, 2009.

[13] Ö. Oyman, "Opportunistic scheduling and spectrum reuse in relay-based cellular networks," IEEE Trans. Wireless Commun., vol. 9, no. 4, pp. 1074-1085, 2010.

[14] Tang, Z., Zhu, Y., Wei, G., Zhu, J., "An OFDMA-based subcarrier and power allocation scheme in wireless cooperative cellular systems." Wireless Personal Communications, vol.48, no.2, pp.277-289, 2008.

[15] Qualcomm Report,“3G/Wi-Fi Seamless Offload.”,March 2010.

[16] IEEE 802.11n Working Group, Wireless LAN Medium Access Control (MAC) and Physical Layer (PHY) Specifications - Amendment 5: Enhancements for Higher Throughput, part 11 standard edition, 2009.

[17] V. Sreng, H. Yanikomeroglu, and D. D. Falconer, "Relay selection strategies in cellular networks with peer-to-peer relaying," IEEE VTC, pp. 1949-1953 October 2003.

[18] 3GPP TS 36.213, "Physical layer procedures," v.8.6.0, March 2009. 